\title{
Quantum computing for quantum tunneling
}

\author{
Steven Abel* \\ Institute for Particle Physics Phenomenology, and Department of Mathematical Sciences, \\ Durham University, Durham DH1 3LE, United Kingdom \\ Nicholas Chancellor $\odot^{\dagger}$ \\ Department of Physics and Durham Newcastle Joint Quantum Centre Durham University, \\ South Road, Durham DH1 3LE, United Kingdom \\ Michael Spannowsky \\ Institute for Particle Physics Phenomenology, Department of Physics, Durham University, \\ Durham DH1 3LE, United Kingdom
}

(Received 30 March 2020; accepted 18 December 2020; published 11 January 2021)

\begin{abstract}
We demonstrate how quantum field theory problems can be practically encoded by using a discretization of the field theory problem into a general Ising model, with the continuous field values being encoded into Ising spin chains. To illustrate the method, and as a simple proof of principle, we use a (hybrid) quantum annealer to recover the correct profile of the thin-wall tunnelling solution. This method is applicable to many nonperturbative problems.
\end{abstract}

DOI: 10.1103/PhysRevD.103.016008

\section{INTRODUCTION}

There has been increasing interest in the possibility of simulating quantum field theory (QFT) on quantum computers [1], with the development of efficient algorithms to compute scattering probabilities in simple theories of scalars and fermions [2-17]. In particular it is known that by latticizing field theories, quantum computers should be able to compute scattering probabilities in QFTs with a run time that is polynomial in the desired precision, and in principle to a precision that is not bounded by the limits of perturbation theory. However a particularly difficult aspect of this programme is the preparation of scattering states [4-6,8,9,14-17], with several works having proposed a hybrid classical/quantum approach to solving this problem [11,17-19]. A complementary approach is to map field theory equations to discrete quantum walks [20-23] which can be simulated on a universal quantum computer.

Certain nonperturbative quantum processes do not suffer from this difficulty, and lend themselves much more readily to study on quantum computers in the short term.

\footnotetext{
s.a.abel@durham.ac.uk

†nicholas.chancellor@gmail.com

\#michael.spannowsky@durham.ac.uk
}

Published by the American Physical Society under the terms of the Creative Commons Attribution 4.0 International license. Further distribution of this work must maintain attribution to the author(s) and the published article's title, journal citation, and DOI. Funded by SCOAP ${ }^{3}$.
These include the tunneling and related processes, which are of fundamental importance for the explanation of quantum mechanical and quantum field theoretical phenomena, for example transmission rates of electron microscopes, first-order phase transitions during baryogenesis, or the potential initiation of stochastic gravitational wave spectra in the early Universe and many more.

Typically in tunneling, the system begins in a false vacuum state that is nondynamical and virtually trivial. The initial state can be very long lived, with tunneling to a lower "true" vacuum state taking place via nonperturbative instanton configurations. The objective of this work is to demonstrate how a field theory problem can be successfully encoded on a quantum spin device, by studying the classic problem of obtaining such tunneling instanton solution for a system stuck in a metastable minimum (a.k.a. false vacuum).

\section{SETUP OF A SIMPLE PROBLEM}

A useful potential to focus on is the following quartic one:

$$
V(\phi)=\frac{\lambda}{8}\left(\phi^{2}-a^{2}\right)^{2}+\frac{\epsilon}{2 a}(\phi-a) .
$$

It is convenient to henceforth remove the extraneous constant term by working with $U(\phi)=V(\phi)-V\left(\phi_{+}\right)$, which has $U\left(\phi_{+}\right)=0$. The resulting potential is shown in Fig. 1. On the left we show the "thick-wall" regime where $\epsilon$ is large. This limit is when the barrier is close to 


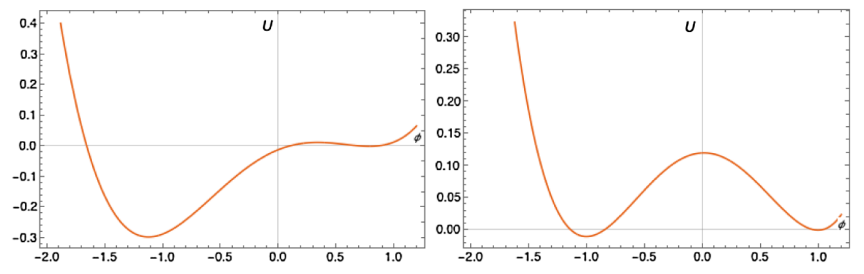

FIG. 1. The thick-wall potential in the left panel (with $\epsilon=0.3$, and true and false minima at $\phi_{-}=-1.12542$ and $\phi_{+}=0.786483$ respectively), and the thin-wall potential in the right panel (with $\epsilon=0.01)$.

disappearing (or has disappeared altogether) and the walls become comparable in size to the bubble itself. For numerics we choose $a=\lambda=1$ and $\epsilon=0.3$. The opposite "thin-wall" regime (for which we choose $\epsilon=0.01$ ) is the limit in which $\epsilon$ is small and is approximately the difference in vacuum energy density between the false and true minima.

We are interested in the situation where the system starts in the false vacuum, and our objective is to determine the rate per unit volume of tunneling out of it. Using the wellknown technique of [24-27], the bubble profile is given by finding a "bounce solution" to the following differential equation:

$$
\frac{d^{2} \phi}{d \rho^{2}}+\frac{c}{\rho} \frac{d \phi}{d \rho}=\frac{\partial U}{\partial \phi}
$$

where in four dimensions, $c$ takes the value 2 or 3 for a finite temperature $O(3)$ symmetric bubble, or a purely quantum tunneling $O(4)$ symmetric instanton, respectively, and where $\rho$ is the radial coordinate of the solution. The required "bounce" is subject to the boundary condition that $d \phi / d \rho=0$ as $\rho \rightarrow 0, \infty$, which determines the starting value $\phi(0)$, which is the field-value at the centre of the radially symmetric bubble or instanton (also called the escape-point). The resulting $\phi(\rho)$ profile for our particular choice of parameters is shown in Fig. 2.

Once such a solution is determined, the tunneling rate per unit volume can be estimated from its classical action:

$$
\Gamma_{4} \sim e^{-S_{4}[\phi]} ; \quad \Gamma_{3} \sim T e^{-S_{3}[\phi] / T},
$$

respectively.

The action can be expressed in simple analytic terms in the two limits. In the thick wall limit the bounce action can be accurately approximated by expanding around the value $\epsilon=\epsilon_{0}$, above which the barrier disappears (i.e., when the discriminant vanishes), which gives a cubic potential about the false vacuum. This critical value corresponds to $\epsilon_{0}=2 \lambda a^{4} / 3 \sqrt{3}$. Then following the rescaling procedure of [27], the tunneling actions for the $O(4)$ and $O(3)$ symmetric solutions can be written in terms of standard actions $S_{4}^{0}=91$ and $S_{3}^{0}=19.4$ :

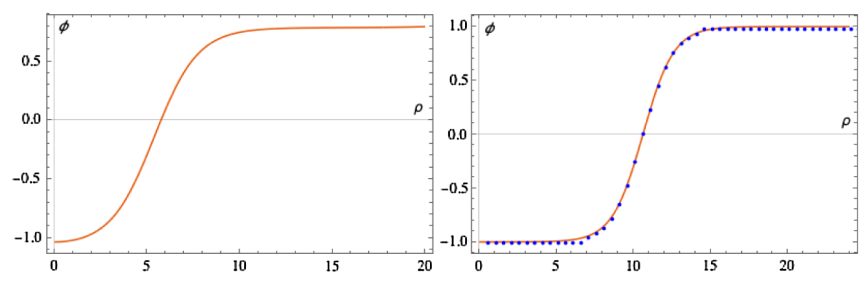

FIG. 2. Solutions for the thick- and thin-wall potentials. Note that in the thick-wall case the solution has to start away from the minimum due to the friction term (in this case $\phi_{e}=-1.03488$ ). The thin-wall solution computed using the hybrid quantumclassical techniques as discussed later is overlaid on the right panel.

$$
S_{4}=\frac{3 \rho}{\lambda} S_{4}^{0} ; \quad S_{3}=\frac{3 a \rho^{3 / 2}}{\lambda^{1 / 2}} S_{3}^{0} .
$$

The thin-wall regime is somewhat easier to study numerically, and semianalytically the actions can be expressed in terms of the action $S_{1}$ for the one-dimensional $c=0$ problem ${ }^{1}$ :

$$
S_{4}=\frac{27 \pi^{2} S_{1}^{4}}{2 \epsilon^{3}} ; \quad S_{3}=\frac{16 \pi^{3} S_{1}^{3}}{3 \epsilon^{2}}
$$

As we stated in the Introduction, the point of our study is not to find these classical instanton solutions for the tunneling per se, which are well known, but rather to demonstrate that the corresponding field-theory configuration can be suitably encoded onto a quantum spin system, for example a quantum annealer. Once we have established this as a working principle, one could even envisage testing for the above behavior directly. Therefore we will in what follows focus on using a quantum device to recover the simple $c=0$ solution required for the thin-wall regime, as a proof of principle. We will therefore set ourselves the task of minimizing the corresponding action integral,

$$
S_{1}=\int_{0}^{\infty} d \rho \frac{1}{2} \dot{\phi}^{2}+U(\phi),
$$

which should yield a solution of the form shown in Fig. 2(b).

\section{ENCODING THE FIELD THEORY}

Let us start with the central problem, which is how to formulate a continuous scalar field theory on a quantum spin system. For this study we will make use of quantum annealers which are a convenient device that is available at the time of writing. But the same method can be used on

\footnotetext{
${ }^{1}$ This is also the energy of the physical "domain wall" solution, but for reasons that will become apparent it would be confusing to use this terminology.
} 
any device that can encode and optimize a generalized Ising model.

A quantum annealer in particular is based on the adiabatic theorem of quantum mechanics, which implies that a physical system will remain in the ground state if a given perturbation acts slowly enough, and if there is a gap between the ground state and the rest of the system's energy spectrum [28].

The crucial form of the Hamiltonian that we are interested in, and that is represented on a quantum annealer is that of a general Ising model, in addition to a timedependent transverse field:

$\mathcal{H}_{\mathrm{QA}}(t)=\sum_{i} \sum_{j} J_{i j} \sigma_{i}^{Z} \sigma_{j}^{Z}+\sum_{i} h_{i} \sigma_{i}^{Z}+\Delta(t) \sum_{i} \sigma_{i}^{X}$,

where $\sigma_{i}^{Z}=\left(\begin{array}{cc}1 & 0 \\ 0 & -1\end{array}\right)\left(\sigma^{Z}|0\rangle=|0\rangle, \sigma^{Z}|1\rangle=-|1\rangle\right)$ is the Pauli $Z$ operator, with the subscript indicating which spin it acts upon, and $\sigma^{X}$ is its friend pointing in the $X$-direction. The gradual decrease of $\Delta(t) \rightarrow 0$ from a large value should drive the system into the ground state of the time-independent part of the Hamiltonian, and this is where we will put the field theory:

$$
\mathcal{H}=\sum_{i} \sum_{j} J_{i j} \sigma_{i}^{Z} \sigma_{j}^{Z}+\sum_{i} h_{i} \sigma_{i}^{Z}
$$

For the annealer to provide a solution to a mathematical problem, e.g., the calculation of $\phi(\rho)$ for Eq. (6), we have to find a mapping such that the expectation value of its Hamiltonian can be identified with its solution, i.e., that it allows in this example to draw a material equivalence:

$$
\phi(\rho) \Leftrightarrow \lim _{\Delta(t) \rightarrow 0}\left\langle\mathcal{H}_{\mathrm{QA}}(t)\right\rangle,
$$

namely that minimizing the Hamiltonian of the quantum annealer, $\mathcal{H}$, is the same as extremizing the field theory action in $\phi$.

It is worth noting that the couplings $J_{i j}$ and $h_{i}$ could also be adiabatically adjusted in the annealing process, and this could ultimately be used to adjust the potential $U(\phi)$ of a system in the quantum annealer so as to observe tunneling, assuming it can be encoded. We will further split the Hamiltonian into three generic pieces, as

$$
\mathcal{H}=\mathcal{H}^{\text {(chain) }}+\mathcal{H}^{(\mathrm{QFT})}+\mathcal{H}^{(\mathrm{BC})} .
$$

Here, $\mathcal{H}^{(\mathrm{QFT})}$ is the Hamiltonian corresponding to the minimization of the action in Eq. (6) and $\mathcal{H}^{(\mathrm{BC})}$ is a Hamiltonian that we add to enforce the boundary conditions. $^{2}$

\footnotetext{
${ }^{2}$ For a classical neural network-based approach to solving Eq. (2) by treating it as an optimization problem see [29].
}

However our first task is to encode continuous field values over a continuous domain, with only the discrete Ising model to hand: this is what $\mathcal{H}^{\text {(chain) }}$ is for. We begin by splitting the radius variable $\rho$ into $M \gg 1$ discrete values and the field value at the $\ell^{\prime}$ th position into $N \gg 1$ discrete values:

$$
\begin{aligned}
\rho_{\ell} & =\ell \nu=\nu \ldots M \nu \\
\phi\left(\rho_{l}\right) & =\phi_{0}+\alpha_{l} \xi=\phi_{0}+\xi \ldots \phi_{0}+N \xi,
\end{aligned}
$$

where in the present context one might for example take a fiducial value $\phi_{0} \approx-a$ and $\xi=2 a / N$, with $M \nu=\Delta \rho$. Thus our Ising interaction $J_{i j}$ is an $(M N) \times(M N)$ matrix, while $h_{i}$ is an $(N M)$-vector.

We must now separate those spins in the annealer that correspond to fields at different values of $\ell$, effectively splitting $J_{i j}$ and $h_{i}$ into $N \times N$ sub-blocks. To do this we will utilize the Ising-chain domain wall representation introduced in [30]. That is for every position $\ell$ we add to the Hamiltonian

$H_{\ell}^{\text {(chain) }}=-\Lambda\left(\sum_{j=1}^{N-1} \sigma_{\ell N+j}^{Z} \sigma_{\ell N+j+1}^{Z}-\sigma_{\ell N+1}^{Z}+\sigma_{\ell N+N}^{Z}\right)$.

As shown in [30], taking $\Lambda$ to be much larger than every other energy scale in the overall Hamiltonian, these terms will constrain the system to remain in the ground subspace of the Hamiltonian, where exactly one spin position, $\alpha_{\ell}$ say, is frustrated for each $\ell$. These states are of the form

$$
|11 \ldots 100 \ldots 0\rangle_{\ell} \Rightarrow \phi\left(\rho_{\ell}\right)=\phi_{0}+\alpha_{\ell} \xi
$$

where in the above the discretized field value is represented by the position $\alpha_{\ell}$ of the frustrated domain wall. Conversely the field value at the $\ell^{\prime}$ th position can be found by making the measurement

$$
\phi\left(\rho_{\ell}\right)=\frac{1}{2} \sum_{j=1}^{N-1}\left(\phi_{0}+j \xi\right)\left\langle\sigma_{\ell N+j+1}^{Z}-\sigma_{\ell N+j}^{Z}\right\rangle,
$$

which only receives a contribution from frustrated spin position with $j=\alpha_{\ell}$. For later, it is useful to note that this is equivalent to

$$
\phi\left(\rho_{\ell}\right)=\phi_{0}+\frac{N \xi}{2}-\frac{\xi}{2} \sum_{j=1}^{N}\left\langle\sigma_{\ell N+j}^{Z}\right\rangle
$$

In terms of $J_{i j}$ and $h_{i}$, adding the full set of Ising-chain Hamiltonians given by Eq. (11) corresponds to 


$$
J_{\ell N+i, m N+j}^{\text {(chain })}=-\frac{\Lambda}{2} \delta_{\ell m} \otimes\left(\begin{array}{cccccc}
0 & 1 & & & & \\
1 & 0 & 1 & & & \\
& 1 & 0 & & & \\
& & & \ddots & & \\
& & & & 0 & 1 \\
& & & & 1 & 0
\end{array}\right)_{i j}
$$

and an $h$ that is independent of $\ell$,

$$
h_{\ell N+j}^{\text {(chain) }}=\Lambda\left(\delta_{j 1}-\delta_{j N}\right) .
$$

This separates the system of spins into blocks of size $N$, each of which represents a position.

Moving on to $\mathcal{H}^{(\mathrm{QFT})}$, the potential is somewhat easier to deal with than the kinetic terms, because it can be encoded entirely in $h_{i}$. This is only to be expected because the $\phi_{\ell}$ are independent of each other in the potential which gives entirely localized contributions to the Hamiltonian. The value of $U\left(\phi\left(\rho_{\ell}\right)\right)$ at each point follows directly from Eq. (13):

$U\left(\phi\left(\rho_{\ell}\right)\right)=\frac{1}{2} \sum_{j=1}^{N-1} U\left(\phi_{0}+j \xi\right)\left\langle\sigma_{\ell N+j+1}^{Z}-\sigma_{\ell N+j}^{Z}\right\rangle$.

This yields an additional contribution to the $h$ which is also independent of $\ell$ : that is for all $\ell$ we have

$h_{N \ell+j}^{(\mathrm{QFT})}= \begin{cases}\frac{\nu}{2}\left(U\left(\phi_{0}+(j-1) \xi\right)-U\left(\phi_{0}+j \xi\right)\right) ; & j<N \\ \frac{\nu}{2} U\left(\phi_{0}+(N-1) \xi\right) ; & j=N .\end{cases}$

Note that in a system with arbitrary $c \neq 0$, we would need to evaluate $h^{(U)} \equiv \int d \rho \rho^{c} U$, so that $h_{\ell N+i}$ would acquire a prefactor of $(\ell \nu)^{c}$.

Up to this point the $M$-factors have been inert and there has been no coupling between the fields at different positions in $\rho_{\ell}$. At this stage the system would simply relax to $M$ decoupled values of $\phi\left(\rho_{\ell}\right)$ that minimize $U$ in either one of its two vacua. This changes once we include the derivatives in the kinetic terms, which contribute to the bilinear interactions, $J$. These terms are discretized in $\rho$ as

$S_{K E} \equiv \int_{0}^{\Delta \rho} d \rho \frac{1}{2} \dot{\phi}^{2}=\lim _{M \rightarrow \infty} \sum_{\ell=1}^{M} \frac{1}{2 \nu}\left(\phi\left(\rho_{\ell+1}\right)-\phi\left(\rho_{\ell}\right)\right)^{2}$,

where $\nu=\Delta \rho / M$ scales so as to keep $\Delta \rho$ constant. Inserting the discrete representation of the field values as well using Eq. (14), we find

$$
\begin{aligned}
S_{K E}= & \sum_{\ell=1}^{M-1} \sum_{i j}^{N-1} \frac{\xi^{2}}{8 \nu}\left[\sigma_{(\ell+1) N+i}^{Z}-\sigma_{\ell N+i}^{Z}\right] \\
& \times\left[\sigma_{(\ell+1) N+j}^{Z}-\sigma_{\ell N+j}^{Z}\right] .
\end{aligned}
$$

Hence the bilinear terms receive the additional contribution:

$$
J_{\ell N+i, m N+j}^{(\mathrm{QFT})}=\frac{\xi^{2}}{8 \nu}\left(\begin{array}{cccccc}
1 & -1 & & & & \\
-1 & 2 & -1 & & & \\
& -1 & 2 & -1 & & \\
& & & \ddots & & \\
& & & -1 & 2 & -1 \\
& & & & -1 & 1
\end{array}\right)_{\ell m}
$$

Now it is the $N \times N$ indices that are inert, because every $i$ couples to every $j$.

Finally we must add terms to enforce a boundary condition. In the $c=0$ case it is sufficient to fix the endpoints of the solution in the two minima (so that, at the risk of confusion, the instanton solution itself approximates a physical domain wall). This can be done by adding a term $\mathcal{H}^{(B C)}=\frac{\Lambda^{\prime}}{2}(\phi(0)+a)^{2}+\frac{\Lambda^{\prime}}{2}\left(\phi\left(\rho_{M}\right)-a\right)^{2}$ with $\Lambda^{\prime}$ being some other large parameter. This is simply an extra contribution to $h$

$h_{N \ell+j}^{(\mathrm{BC})}= \begin{cases}-\Lambda^{\prime}\left(\phi_{0}+j \xi+a\right) ; & \ell=1, \quad \forall j \\ -\Lambda^{\prime}\left(\phi_{0}+j \xi-a\right) ; & \ell=M-1, \quad \forall j .\end{cases}$

Together with Eqs. (15), (16), and (21), this completes the encoding of the field theory problem of Eq. (6).

\section{EXTENSION TO $U(1)$ STRING}

Extension to the case of multiple fields and multiple dimensions would be straightforward although current technology does not make it very feasible. As an example we will consider the $U(1)$ topological string in two dimensions. Consider the following energy integral

$$
H_{U(1)}=\int_{0}^{\infty} d^{2} x \frac{1}{2} \nabla \phi_{a} \cdot \nabla \phi^{a}+U\left(\phi_{a}\right)
$$

where $a=0,1$ are internal indices, and where

$$
U\left(\phi_{a}\right)=\frac{\lambda}{8}\left(\phi_{0}^{2}+\phi_{1}^{2}-v^{2}\right)^{2} .
$$

Obviously this two-dimensional theory has a $U(1) \sim$ $S O(2)$ rotational symmetry, and it has a topologically stable $S O(2)$ symmetric configuration in $\mathbb{R}^{2}$, the $U(1)$ 
string. In principle this could be determined exactly as before as a $c=1$ bubble, with $\rho$ being the radius. However let us pretend that we do not know about the rotational symmetry and solve it as a 2D problem with two fields. (It is probably also worth mentioning that strictly speaking we will be finding the solution for a string in a finite box, which is also not rotationally symmetric).

We first need to go back to the beginning and decide how we are going to assign the dimensions and fields to Ising spins. The simplest is to vectorize the indices $\ell \rightarrow \ell_{\mu=0 \ldots d-1}$ and $i \rightarrow i_{a=0 \ldots n-1}$, where $d$ is the number of space dimensions and $n$ is the number of fields. Obviously the indices are then flattened to fit into the bilinear Ising model; for example the field values at a single point $\phi_{a}\left(x_{\mu}\right)$ can be represented by the spins at the $n$ positions

$$
\left\{i_{a}\right\} \equiv\left(n \ell_{\mu} M^{\mu-1}+a\right) N+i_{a} ; \quad a=0 \ldots n-1 .
$$

That is, the position $\ell_{\mu}$ is identified by the multiple of $n$ with an integer in base- $M$, i.e., $\ell=n \ell_{\mu} M^{\mu-1}$ with each $\ell_{\mu}$ yielding $n \times N$ values of $i_{a}$. The Ising model scales as an $n M^{d} N \times n M^{d} N$ matrix.

Clearly the Ising chain coefficients are for a field value at the $\ell_{\mu}$ 'th position, hence Eq. (15) still has a prefactor $\delta_{\ell m}$, and both Eqs. (15) and (16) are for all $i_{a} j_{b}$ indices: for the chain $J$ and $h$ we have

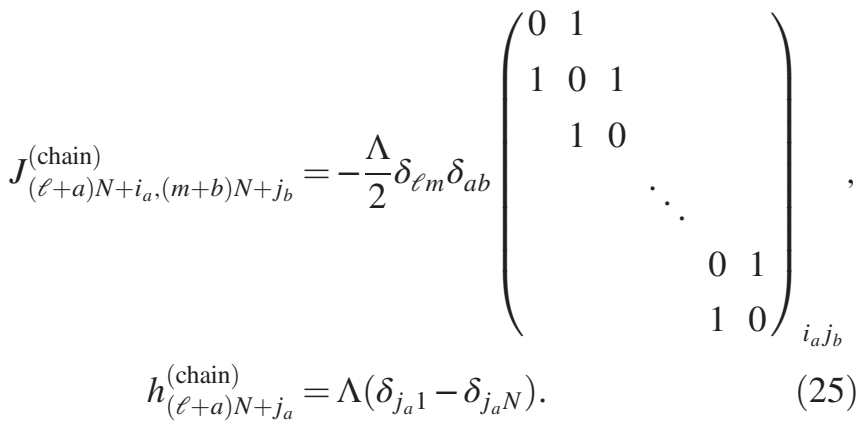

To encode the QFT, we begin with the value of $U\left(\phi_{a}\left(x_{\ell}^{\mu}\right)\right)$ at each point which follows again from Eq. (13). For the $U(1)$ case, we can specialize to the 2 -field case (or more generally interactions that involve at most two fields). The simplest way to incorporate such terms is as contributions to $J$ : denoting the fiducial field values by $\hat{\phi}_{a}$, according to Eq. (13) we have

$$
\begin{aligned}
U= & \frac{1}{4} \sum_{j_{0}, j_{1}=1}^{N} U\left(\hat{\phi}_{0}+j_{0} \xi, \hat{\phi}_{1}+j_{1} \xi\right)\left\langle\sigma_{\ell N+j_{0}+1}^{Z}-\sigma_{\ell N+j_{0}}^{Z}\right\rangle \\
& \times\left\langle\sigma_{(\ell+1) N+j_{1}+1}^{Z}-\sigma_{(\ell+1) N+j_{1}}^{Z}\right\rangle
\end{aligned}
$$

which translates into

$$
\begin{aligned}
& J_{(\ell+a) N+i_{a},(m+b) N+j_{b}}^{(U)} \\
& =\frac{\nu^{2}}{8} \delta_{\ell m}\left(1-\delta_{a b}\right) U\left(\hat{\phi}_{0}+j_{0} \xi, \hat{\phi}_{1}+j_{1} \xi\right) \\
& \quad+U\left(\hat{\phi}_{0}+\left(j_{0}-1\right) \xi, \hat{\phi}_{1}+\left(j_{1}-1\right) \xi\right) \\
& \quad-U\left(\hat{\phi}_{0}+j_{0} \xi, \hat{\phi}_{1}+\left(j_{1}-1\right) \xi\right) \\
& \quad-U\left(\hat{\phi}_{0}+\left(j_{0}-1\right) \xi, \hat{\phi}_{1}+j_{1} \xi\right),
\end{aligned}
$$

regardless of $\ell$. Note that it is possible to encode couplings between three or more fields, but at the expense of introducing more qubits [31,32]. Meanwhile the Laplacian is generated by

$$
\begin{aligned}
& J_{(\ell+a) N+i_{a},(m+b) N+j_{b}}^{(\Delta)} \\
& \quad=\frac{\xi^{2}}{8} \delta_{a b} \sum_{\mu=0}^{d-1} 2 \delta_{\ell m}-\delta_{\ell\left(m+n M^{\mu}\right)}-\delta_{\left(\ell+n M^{\mu}\right) m} .
\end{aligned}
$$

In order to for example describe the 2D QFT inside the square $x_{0} \equiv x \in\left(0, x_{M}\right)$ and $x_{1} \equiv y \in\left(0, y_{M}\right)$, we have $\ell \equiv 4 \ell_{1}+2 \ell_{0}$. Let us choose boundary conditions such that the field maps to the square boundary pointing horizontally or vertically. This can be implemented by adding terms $\mathcal{H}^{(B C)}$ such that

$$
\begin{aligned}
\frac{2}{\Lambda^{\prime}} \mathcal{H}^{(B C)}= & \left(\phi_{0}(0, y)+v\right)^{2}+\left(\phi_{0}\left(x_{M}, y\right)-v\right)^{2} \\
& +\left(\phi_{1}(x, 0)+v\right)^{2}+\left(\phi_{1}\left(x, y_{M}\right)-v\right)^{2} .
\end{aligned}
$$

In a straightforward adaption of the 1D case, these are imposed by

$$
\begin{aligned}
-\frac{1}{\Lambda^{\prime}} h_{\ell N+j_{0}}^{(\mathrm{BC})} \stackrel{\forall \ell_{1}, j_{0}}{=} \begin{cases}\hat{\phi}_{0}+j_{0} \xi+v ; & \ell_{0}=1, \\
\hat{\phi}_{0}+j_{0} \xi-v ; & \ell_{0}=M-1,\end{cases} \\
-\frac{1}{\Lambda^{\prime}} h_{(\ell+1) N+j_{1}}^{(\mathrm{BC})} \stackrel{\forall \ell_{0}, j_{1}}{=} \begin{cases}\hat{\phi}_{1}+j_{1} \xi+v ; & \ell_{1}=1, \\
\hat{\phi}_{1}+j_{1} \xi-v ; & \ell_{1}=M-1 .\end{cases}
\end{aligned}
$$

In this manner, the lifting to arbitrary numbers of dimensions becomes very straightforward. As mentioned above the discretization of space-time dimension makes this currently relatively costly as the size of Ising model increases as $N M^{d}$, where $N$ is the number of field values and $M$ the number of discretized space-points. (Currently in 2 dimensions one could still only manage roughly 20 space time points and 12 field values even if one were able to utilize every available qubit of the current state-of-the art which is a 5000 qubit machine, and even if it were perfectly connected). 


\section{IMPLEMENTATION ON A QUANTUM SPIN DEVICE AS A GENERAL ISING MODEL}

In Sec. III we have devised a method which encodes the problem of finding a solution to a quantum field theoretical problem, i.e., of finding a solution to Eq. (6), into finding the ground state of the Hamiltonian of an Ising model. The latter can then be given an interpretation as the solution to Eq. (6) through Eq. (12), for each $\rho_{l}$ with $l \in[1, \ldots, M]$. To show that our approach is valid and converges to the correct solution $\phi(\rho)$, we now implement the method onto various annealing samplers, as provided by D-Wave [33].

As discussed quantum annealers [28,34] perform continuous time quantum computations in Ising systems, so they are extremely well-suited to the problem at hand (although our discussion could ultimately be adapted to gate-model quantum computers as well) [35-47]. In contrast with the quantum-gate devices, they are already quite large, 2048 qubits in the current generation, with work ongoing to develop much more connected 5,000 qubit machines. D-Wave devices have been able successfully to simulate condensed matter systems, sometimes showing advantages over classical counterparts [48-50].

The quantum states are characterized by $N M$-tuples of the form $|11 \ldots 100 \ldots 0\rangle$ and the Hilbert space of the Ising model is therefore $2^{N M}$ dimensional. Sampling such a large vector space classically, with an exact sampler, while calculating the expectation value $\langle\mathcal{H}\rangle$ for each state quickly becomes a computationally prohibitive task for $N M \gg 20$. Conversely, a discretization with $N M \lesssim 20$ cannot give a reasonable approximation for the derivatives of Eq. (19).

Yet, not unlike protein-folding, in which a unique ground state is selected from an estimated number of $3^{300}$ so-called conformations within microseconds (known as Levinthal's paradox [51]), a quantum annealer can in principle find a ground state of a Hamiltonian acting on a highly complex Hilbert space on a similar time scale, assuming there is a gap between the ground state and the other states of the system.

While the next generation of annealing processors will have approximately 5,000 qubits, they will have limited connectivity [52]. Therefore in order to accommodate the more general Ising model required for our encoding, we resorted to a hybrid asynchronous decomposition sampler (the Kerberos solver [53-55]), which can solve problems of complex structure and size. To find the ground state efficiently, it applies in parallel classical tabu search algorithms, simulated annealing and D-Wave subproblem sampling on variables that have high-energy impact. Using this method we calculate the solution $\phi(\rho)$ to Eq. (6) for $N=M=50$ in Fig. 2(b). We observe that the discretized field theory has minimized at the expected tunneling solution, and the field theory and its Hamiltonian are successfully encoded in the Ising model.

\section{CONCLUSION}

We conclude that near-term applications of quantum devices already allow the implementation of a wide class of scalar field theories. By encoding them as an Ising model, as described in Sec. III, we have been able to find tunneling solutions on a D-Wave quantum annealer, which match very precisely with the classical instanton solution. It is important to appreciate that our end result is essentially an experimental one: it is a genuine field theory relaxing to the predicted solution. Thus the method is a novel, highly flexible and possibly transformative way to probe nonperturbative aspects of a wide class of field theories.

Our method has the potential to consider theories and processes that are not easy to simulate and cannot be treated analytically either. The variety of theory that can be studied in this laboratory appears to be limited mainly by the number and connectivity of the qubits in the generalized Ising model. For example the Ising encoding of fermions and of gauge theories is a longstanding topic (see for example [56,57]). It is important to realize that these encodings represent genuine condensed matter field theories constructed from a set of aligned quantum spins. In this sense although the toy problem discussed here is an optimization that can be done classically, depending on the set-up these configurations can be morally different from a simulation. For example the system can support actual dynamical quantum tunneling rather than a simulation of it [58].

\section{ACKNOWLEDGMENTS}

N.C. was funded by UK Research and Innovation (UKRI) fellowship No. EP/S00114X/1.
[1] R. P. Feynman, Simulating physics with computers, Int. J. Theor. Phys. 21, 467 (1982); 21, 923 (1981).

[2] C. Zalka, Efficient simulation of quantum systems by quantum computers, Proc. R. Soc. A 454, 313 (1998).

[3] S. P. Jordan, K. S. M. Lee, and J. Preskill, Quantum algorithms for quantum field theories, Science 336, 1130 (2012).
[4] S. P. Jordan, K. S. M. Lee, and J. Preskill, Quantum computation of scattering in scalar quantum field theories, Quantum Inf. Comput. 14, 1014 (2014).

[5] L. García-Álvarez, J. Casanova, A. Mezzacapo, I. L. Egusquiza, L. Lamata, G. Romero, and E. Solano, Fermion-Fermion Scattering in Quantum Field Theory with 
Superconducting Circuits, Phys. Rev. Lett. 114, 070502 (2015).

[6] S. P. Jordan, K. S. M. Lee, and J. Preskill, Quantum algorithms for fermionic quantum field theories, arXiv: 1404.7115 .

[7] M. E. Beverland, O. Buerschaper, R. Koenig, F. Pastawski, J. Preskill, and S. Sijher, Protected gates for topological quantum field theories, J. Math. Phys. (N.Y.) 57, 022201 (2016).

[8] S. P. Jordan, H. Krovi, K. S. M. Lee, and J. Preskill, BQPcompleteness of scattering in scalar quantum field theory, Quantum 2, 44 (2018).

[9] A. Hamed Moosavian and S. Jordan, Faster quantum algorithm to simulate fermionic quantum field theory, Phys. Rev. A 98, 012332 (2018).

[10] J. Preskill, Simulating quantum field theory with a quantum computer, Proc. Sci., LATTICE2018 (2018) 024 [arXiv: 1811.10085].

[11] H. Lamm and S. Lawrence, Simulation of Nonequilibrium Dynamics on a Quantum Computer, Phys. Rev. Lett. 121, 170501 (2018).

[12] K. Yeter-Aydeniz, E. F. Dumitrescu, A. J. McCaskey, R. S. Bennink, R. C. Pooser, and G. Siopsis, Scalar quantum field theories as a benchmark for near-term quantum computers, Phys. Rev. A 99, 032306 (2019).

[13] C. W. Bauer, W. A. De Jong, B. Nachman, and D. Provasoli, A quantum algorithm for high energy physics simulations, arXiv:1904.03196.

[14] A. H. Moosavian, J. R. Garrison, and S. P. Jordan, Site-bysite quantum state preparation algorithm for preparing vacua of fermionic lattice field theories, arXiv:1911.03505.

[15] E. Gustafson, P. Dreher, Z. Hang, and Y. Meurice, Real time evolution of a one-dimensional field theory on a 20 qubit machine, arXiv:1910.09478.

[16] N. Klco and M. J. Savage, Systematically localizable operators for quantum simulations of quantum field theories, Phys. Rev. A 102, 012619 (2020).

[17] S. Harmalkar, H. Lamm, and S. Lawrence (NuQS Collaboration), Quantum simulation of field theories without state preparation, arXiv:2001.11490.

[18] A. Y. Wei, P. Naik, A. W. Harrow, and J. Thaler, Quantum algorithms for jet clustering, Phys. Rev. D 101, 094015 (2020).

[19] K. T. Matchev, P. Shyamsundar, and J. Smolinsky, A quantum algorithm for model independent searches for new physics, arXiv:2003.02181.

[20] P. Arrighi, G. D. Molfetta, I. Márquez-Mártin, and A. Pérez, The Dirac equation as a quantum walk over the honeycomb and triangular lattices, Phys. Rev. A 97, 062111 (2018).

[21] I. Márquez-Mártin, P. Arnault, G. D. Molfetta, and A. Pérez, Electromagnetic lattice gauge invariance in twodimensional discrete-time quantum walks, Phys. Rev. A 98, 032333 (2018).

[22] G. Jay, F. Debbasch, and J. B. Wang, Dirac quantum walks on triangular and honeycomb lattices, Phys. Rev. A 99, 032113 (2019).

[23] G. D. Molfetta and P. Arrighi, A quantum walk with both a continuous-time limit and a continuous-spacetime limit, Quantum Inf. Process. 19, 47 (2020).
[24] S. R. Coleman, The fate of the false vacuum. 1. Semiclassical theory, Phys. Rev. D 15, 2929 (1977); Erratum, Phys. Rev. D 16, 1248 (1977).

[25] C. G. Callan, Jr. and S. R. Coleman, The fate of the false vacuum. 2. First quantum corrections, Phys. Rev. D 16, 1762 (1977).

[26] S. Coleman, Aspects of Symmetry: Selected Erice Lectures (Cambridge University Press, Cambridge, England, 1988).

[27] A. D. Linde, Decay of the false vacuum at finite temperature, Nucl. Phys. B216, 421 (1983); Erratum, Nucl. Phys. 223, 544 (1983).

[28] E. Farhi, J. Goldstone, S. Gutmann, and M. Sipser, Quantum computation by adiabatic evolution, arXiv:quant-ph/ 0001106.

[29] M. L. Piscopo, M. Spannowsky, and P. Waite, Solving differential equations with neural networks: Applications to the calculation of cosmological phase transitions, Phys. Rev. D 100, 016002 (2019).

[30] N. Chancellor, Domain wall encoding of discrete variables for quantum annealing and QAOA, Quantum Sci. Technol. 4, 045004 (2019).

[31] A. B. Dodds, V. Kendon, C. S. Adams, and N. Chancellor, Practical designs for permutation-symmetric problem Hamiltonians on hypercubes, Phys. Rev. A 100, 032320 (2019).

[32] N. Chancellor, S. Zohren, and P. A. Warburton, Circuit design for multi-body interactions in superconducting quantum annealing systems with applications to a scalable architecture, npj Quantum Inf. 3 (2017).

[33] D-Wave, D-wave systems inc., website. http://www .dwavesys.com/, 2019 (Accessed: 2019-05-03).

[34] H. Neven, V. S. Denchev, M. Drew-Brook, J. Zhang, W. G. Macready, and G. Rose, Nips 2009 demonstration: Binary classification using hardware implementation of quantum annealing, 2009.

[35] A. B. Finilla, M. A. Gomez, C. Sebenik, and J. D. Doll, Quantum annealing: A new method for minimizing multidimensional functions, Chem. Phys. Lett. 219, 343 (1994).

[36] T. Kadowaki and H. Nishimori, Quantum annealing in the transverse Ising model, Phys. Rev. E 58, 5355 (1998).

[37] J. Brooke, D. Bitko, T. F. Rosenbaum, and G. Aeppli, Quantum annealing of a disordered magnet, Science 284, 779 (1999).

[38] N. G. Dickson et al., Thermally assisted quantum annealing of a 16-qubit problem, Nat. Commun. 4, 1903 (2013).

[39] T. Lanting et al., Entanglement in a Quantum Annealing Processor, Phys. Rev. X 4, 021041 (2014).

[40] T. Albash, W. Vinci, A. Mishra, P. A. Warburton, and D. A. Lidar, Consistency tests of classical and quantum models for a quantum annealer, Phys. Rev. A 91, 042314 (2015).

[41] T. Albash and D. A. Lidar, Adiabatic quantum computing, Rev. Mod. Phys. 90, 015002 (2018).

[42] S. Boixo, V. N. Smelyanskiy, A. Shabani, S. V. Isakov, M. Dykman, V. S. Denchev, M. H. Amin, A. Y. Smirnov, M. Mohseni, and H. Neven, Computational multiqubit tunnelling in programmable quantum annealers, Nat. Commun. 7, 10327 (2016).

[43] N. Chancellor, S. Szoke, W. Vinci, G. Aeppli, and P. A. Warburton, Maximum-entropy inference with a programmable annealer, Sci. Rep. 6, 22318 (2016). 
[44] M. Benedetti, J. Realpe-Gómez, R. Biswas, and A. Perdomo-Ortiz, Estimation of effective temperatures in quantum annealers for sampling applications: A case study with possible applications in deep learning, Phys. Rev. A 94, 022308 (2016).

[45] S. Muthukrishnan, T. Albash, and D. A. Lidar, Tunneling and Speedup in Quantum Optimization for PermutationSymmetric Problems, Phys. Rev. X 6, 031010 (2016).

[46] T. Lanting, The D-wave $2000 \mathrm{Q}$ processor, in Presented at AQC 2017.

[47] N. Chancellor, Modernizing quantum annealing using local searches, New J. Phys. 19, 023024 (2017).

[48] R. Harris et al., Phase transitions in a programmable quantum spin glass simulator, Science 361, 162 (2018).

[49] A. D. King et al., Observation of topological phenomena in a programmable lattice of 1,800 qubits, Nature (London) 560, 456 (2018).

[50] A. D. King et al., Scaling advantage in quantum simulation of geometrically frustrated magnets, arXiv:1911.03446.

[51] C. Levinthal, How to fold graciously, in Mossbauer Spectroscopy in Biological Systems: Proceedings (1969),
Vol. 67, pp. 22-26, http://www-miller.ch.cam.ac.uk/ levinthal/levinthal.html.

[52] K. Boothby, P. Bunyk, J. Raymond, and A. Roy, Nextgeneration topology of d-wave quantum processors, arXiv: 2003.00133.

[53] Z. Bian, F. Chudak, R. Israel, B. Lackey, W. Macready, and A. Roy, Discrete optimization using quantum annealing on sparse Ising models, Front. Phys. 2 (2014).

[54] Z. Bian, F. Chudak, R. Israel, B. Lackey, W. Macready, and A. Roy, Mapping optimization problems to quantum annealing with application to fault diagnosis, Front. ICT 3 (2016).

[55] D-Wave Systems Inc., dwave-hybrid documentation, accessed: 15 (2020), https://docs.ocean.dwavesys.com/en/ latest/docs_hybrid/sdk_index.html.

[56] J. Alicea, New directions in the pursuit of Majorana fermions in solid state systems, Rep. Prog. Phys. 75, 076501 (2012).

[57] J. B. Kogut, An introduction to lattice gauge theory and spin systems, Rev. Mod. Phys. 51, 659 (1979).

[58] S. Abel and M. Spannowsky, Observing the fate of the false vacuum with a quantum laboratory, arXiv:2006.06003. 